\title{
Line sepsis rates are significantly improved in parenteral nutrition patients by having a Nutrition Nurse
}

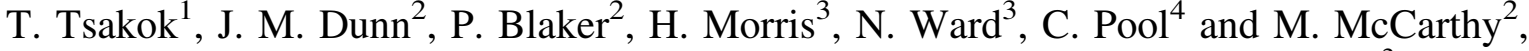 \\ ${ }^{1}$ Academic Foundation Program, Guy's and St Thomas' NHS Foundation Trust, London SE1 7EH, UK, ${ }^{2}$ Gastroenterology \\ Department, Guy's and St Thomas' NHS Foundation Trust, London SE1 7EH, UK, ${ }^{3}$ Nutrition and Dietetics Department, \\ Guy's and St Thomas' NHS Foundation Trust, London SE1 7EH, UK and ${ }^{4}$ Pharmacy Department, Guy's and St Thomas' \\ NHS Foundation Trust, London SE1 7EH, UK
}

Recent UK NCEPOD ${ }^{(1)}$ data highlights catheter line sepsis as a major complication of Parenteral Nutrition (PN). We hypothesised that appointing a specialist Nutrition Nurse may decrease line sepsis rates by improving education of ward staff on line care. Our aim was to evaluate line complications in patients treated at Guy's \& St Thomas' Hospitals before and after this intervention.

We conducted a prospective dual-centre cohort study of patients started on PN over two separate 12-month periods (2005 and 2010). Clinical data was collected via a pro forma and microbiology data was collected via Electronic Patient Records. 221 patients were recruited (141 in 2010, 80 in 2005). From the 2010 cohort, 90 patients were ward-based, whilst 57 were on ICU. Duration of PN ranged from 1-171 days (mean 17 days). Access included true central lines (65\%) or peripherally-inserted central (PICC) lines (35\%). Use of PICC lines had increased since 2005 (16\% PICC lines vs. $84 \%$ true central lines).

In the 2010 study group, incidence of line sepsis was $11 \%$ - this was much improved from $31 \%$ in 2005 (Fisher's exact test $\mathrm{P}=0.0002)$. In $9 / 15$ cases, line sepsis was confirmed by positive blood/line tip culture. Line complications were markedly more likely to occur in patients on wards than on ICU ( $34 \%$ vs. $2 \%$, Fisher's exact test $P<0.0001)$. By contrast, rates of confirmed line sepsis were not significantly influenced by line type (Fisher's exact test $\mathrm{P}=0.5$ ).

This dual-centre audit demonstrates a significant reduction in catheter-related sepsis between 2005 (31\%) and 2010 (11\%). This is likely due to the introduction of a Nutrition Nurse to facilitate conscientious monitoring and line care in PN patients. The discrepancy in line complication rates between wards and ICU indicates that there remains scope to optimise line care on wards. Increased use of peripherally-inserted feeding lines has had no significant impact on line sepsis rates.

1. Stewart JAD, Mason DG, Smith N, Protopapa K \& Mason M A Mixed Bag - an enquiry into the care of hospital patients receiving parenteral nutrition. NCEPOD 2010. 